University of Nebraska - Lincoln

DigitalCommons@University of Nebraska - Lincoln

Publications from USDA-ARS / UNL Faculty

U.S. Department of Agriculture: Agricultural

Research Service, Lincoln, Nebraska

2011

Evaluation of a wireless infrared thermometer with a narrow field of view

Susan A. O'Shaughnessy

USDA-ARS, Susan.OShaughnessy@ars.usda.gov

Martin A. Hebel

Southern Illinois University, mhebel@siu.edu

Steven R. Evett

USDA-ARS, steve.evett@ars.usda.gov

Paul D. Colaizzi

USDA-ARS, Paul.Colaizzi@ARS.USDA.GOV

Follow this and additional works at: https://digitalcommons.unl.edu/usdaarsfacpub

O'Shaughnessy, Susan A.; Hebel, Martin A.; Evett, Steven R.; and Colaizzi, Paul D., "Evaluation of a wireless infrared thermometer with a narrow field of view" (2011). Publications from USDA-ARS / UNL Faculty. 1851.

https://digitalcommons.unl.edu/usdaarsfacpub/1851

This Article is brought to you for free and open access by the U.S. Department of Agriculture: Agricultural Research Service, Lincoln, Nebraska at DigitalCommons@University of Nebraska - Lincoln. It has been accepted for inclusion in Publications from USDA-ARS / UNL Faculty by an authorized administrator of DigitalCommons@University of Nebraska - Lincoln. 


\title{
Evaluation of a wireless infrared thermometer with a narrow field of view
}

\author{
Susan A. O'Shaughnessy ${ }^{\mathrm{a}, *}$, Martin A. Hebel ${ }^{\mathrm{b}}$, Steve R. Evett ${ }^{\mathrm{a}}$, Paul D. Colaizzi ${ }^{\mathrm{a}}$ \\ a United States Department of Agriculture-Agricultural Research Service, Conservation and Production Laboratory, Bushland, TX 79012, USA \\ b Southern Illinois University, Information Systems and Applied Technologies, Carbondale, 62901 IL, USA
}

\section{A R T I C L E I N F O}

\section{Article history:}

Received 19 September 2010

Received in revised form

21 December 2010

Accepted 22 December 2010

\section{Keywords:}

Infrared thermometers

Irrigation water management

Precision agriculture

Remote sensing

Wireless sensors

\begin{abstract}
A B S T R A C T
Many agricultural studies rely on infrared sensors for remote measurement of surface temperatures for crop status monitoring and estimating sensible and latent heat fluxes. Historically, applications for these non-contact thermometers employed the use of hand-held or stationary industrial infrared thermometers (IRTs) wired to data loggers. Wireless sensors in agricultural applications are a practical alternative, but the availability of low cost wireless IRTs is limited. In this study, we designed prototype narrow $\left(10^{\circ}\right)$ field of view wireless infrared sensor modules and evaluated the performance of the IRT sensor by comparing temperature readings of an object $\left(T_{o b j}\right)$ against a blackbody calibrator in a controlled temperature room at ambient temperatures of $15^{\circ} \mathrm{C}, 25^{\circ} \mathrm{C}, 35^{\circ} \mathrm{C}$, and $45^{\circ} \mathrm{C}$. Additional comparative readings were taken over plant and soil samples alongside a hand-held IRT and over an isothermal target in the outdoors next to a wired IRT. The average root mean square error (RMSE) and mean absolute error (MAE) between the collected IRT object temperature readings and the blackbody target ranged between 0.10 and $0.79^{\circ} \mathrm{C}$. The wireless IRT readings also compared well with the hand-held IRT and wired industrial IRT. Additional tests performed to investigate the influence of direct radiation on IRT measurements indicated that housing the sensor in white polyvinyl chloride provided ample shielding for the self-compensating circuitry of the IR detector. The relatively low cost of the wireless IRT modules and repeatable measurements against a blackbody calibrator and commercial IR thermometers demonstrated that these wireless prototypes have the potential to provide accurate surface radiometric temperature readings in outdoor applications. Further studies are needed to thoroughly test radio frequency communication and power consumption characteristics in an outdoor setting.
\end{abstract}

Published by Elsevier B.V.

\section{Introduction}

Infrared thermometers (IRTs) have been widely used in agricultural research to remotely measure surface temperatures and develop vegetation stress indexes (Jackson et al., 1981; Colaizzi et al., 2003), monitor crop canopy temperatures and schedule irrigations based on plant feedback (Jones, 2004; Peters and Evett, 2007), predict crop yields (Pinter et al., 1983; Irmak et al., 2000; Ajayi and Olufayo, 2004), or estimate sensible and latent heat fluxes (e.g. Kustas and Norman, 1997; Kalma and Jupp, 1990). Historically, these applications employed the use of stationary industrial IRTs wired to data loggers. These sensors can be expensive, cumbersome, and impractical for commercial on-farm applications. Wireless IRTs in agricultural applications are a more practical alternative, but their availability is limited. Economical radiation thermometry detectors with both analog and digital outputs are readily available on the market and have been used in the auto-

\footnotetext{
* Corresponding author. Tel.: +1 806356 5770; fax: +1 8063565770.

E-mail address: Susan.OShaughnessy@ars.usda.gov (S.A. O’Shaughnessy).
}

mobile and medical industry, but there are few studies concerning these sensors in agricultural settings.

A specific example of a wireless IRT module that has been evaluated in agricultural applications includes the low-cost wireless IRT work by Mahan and Yeater (2008) and Mahan et al. (2010). Although this IRT had a wide (1:1) field of view (FOV) and required the targets of interest be located near the IRT (approximately within $10 \mathrm{~cm}$ ), it was reported to be similar in accuracy to an industrial-quality sensor over a $13-35^{\circ} \mathrm{C}$ range of target temperatures in an outdoor environment. These IRTs are now available on the market and compared well with measurements from a commercial wired IRT when placed in a cotton field in Australia and monitored over a four-month period (Mahan et al., 2010). In separate work, O'Shaughnessy and Evett (2010) constructed a wireless IRT sensor by interfacing a commercial narrow field of view IRT (IRT/c.5-T-80F/27C, Exergen, ${ }^{1}$ Inc., Watertown, Mass.) with an off-

\footnotetext{
1 Mention of trade names or commercial products in this paper is solely for the purpose of providing specific information and does not imply recommendation or endorsement by the U.S. Department of Agriculture.
} 
the-shelf radio frequency (RF) module using the open ZigBee (IEEE Std. 802.15.4, 2007) communication protocol (O'Shaughnessy and Evett, 2007). The Zigbee protocol is characterized by a slow data transfer rate $(250 \mathrm{kbp} @ 2.4 \mathrm{GHz}$ ), power management to ensure low power consumption, and is optimized for short-duty cycle applications which are critical for power conservation. An overview of sensor technologies and standards for wireless communications in agriculture are summarized by Wang et al. (2006) and will not be discussed in this manuscript. A network of these wireless IRTs was created by mounting the IRTs onto a center pivot lateral for monitoring crop canopy temperatures using methods described by O'Shaughnessy and Evett (2010). Temperature data were transmitted to an embedded computer at the pivot point and used in an algorithm for automatic irrigation scheduling. The development of reliable wireless network systems that transmit data to a base station computer or nearby data logging device increases the convenience and flexibility of sensor use (ease of mobility and reduced maintenance of wiring) for researchers and producers. The use of an open communication protocol will allow incorporation of sensors developed by various manufacturers into sensor network systems without the added expense of specialized receivers and additional interface software at the base station level. Undoubtedly, dependable wireless networks of infrared thermometers that are capable of being integrated into agricultural systems for crop monitoring and machine control will also aid in the commercialization of precision agricultural applications.

As with any scientific measurement, there is an inevitable amount of uncertainty when assessing measurements made by sensors; these uncertainties must be addressed to minimize error (Taylor, 1997). Infrared thermometers measure thermal radiation emitted by an object. All objects at temperatures greater than absolute zero radiate energy in the infrared range (Kaplan, 2007).

Thermal radiation emitted from an object is proportional to the fourth power of the absolute temperature of an object's surface:

$q=\sigma \varepsilon T^{4}$

where $q$ is the emitted radiation, $\sigma$ is Stefan Boltzmann's constant $\left(5.67 \times 10^{-8} \mathrm{~W} \mathrm{~m}^{-2} \mathrm{~K}^{-4}\right), \varepsilon$ is emissivity, and $T$ is absolute surface temperature of the object $(\mathrm{K})$. Radiation emitted from a target is a function of emissivity and surface temperature. However, the output of a conventional IRT depends on the temperature difference between the detector and target and the sensor and is a weighted average of the radiating components within its FOV (Wanjura and Upchurch, 2001). An IRT senses not only the emitted radiation but also the reflected thermal radiation from the target surface and the radiation from the internal surface of the IRT (Baker et al., 2001). The degree to which an IRT is sensitive to reflected and internal radiation is affected by the FOV (Brewster, 1992). Uncertainties associated with infrared thermometer signals are a combination of the effects of ambient temperature, the alignment of the thermometer, detector noise and non-linearity (Shimizu and Ishii, 2008), calibration shifts, the inability to eliminate or properly account for the influence of detector temperature on the measurement (Baker et al., 2001), true target emissivity values, and background radiation.

Our interest in a narrow FOV wireless IRT is to provide the ability to accurately monitor row crops using sensor networks located on moving irrigation systems (Sadler et al., 2002; Peters and Evett, 2004). A sensor with a narrow FOV can be mounted on a pivot lateral and maintained at a stationary height. This is in contrast to a wider FOV sensor located on a mast in a field which requires periodically adjusting its height above the crop canopy as it grows. An IRT with a $10^{\circ}$ FOV, mounted on a pivot lateral ( $\sim 5.1 \mathrm{~m}$ above the ground), looking slightly forward of the drop hoses at an oblique angle to the vertical mast and at a $45^{\circ}$ (in the horizontal plane) from the edge of a treatment plot, will have a resolve of approximately $0.7 \mathrm{~m}^{2}$ of crop surface. The surface can be approximated as an elliptical foot print with the vertical axis estimated as:

$b=h \tan (\alpha / 2)$

where $h$ is the height of the pivot lateral above the ground, $\alpha$ is the IRT's FOV, and $L_{i}$ is the path length from the IRT to the canopy. The horizontal axis, $a$, can be approximated as:

$a=\frac{\sqrt{L_{2}^{3}+L_{3}^{2}-2 L_{2} L_{3} \cos (\alpha / 2)}}{2}$

and the area of the ellipse:

Area $=a b \Pi$

Positioning of an IRT in this manner will help reduce the amount of soil viewed by the sensor (Peters and Evett, 2004) when canopy is less than full and help to reduce sun angle effects by averaging the temperature readings of shaded and unshaded sides of the canopy (Fig. 1).

A fundamental difficulty with IR thermometry is that all objects emit thermal radiation, including the internal surface of an IR detector. Each type of detector, whether it is thermal, photon, or pyroelectric is strongly affected by ambient temperature changes which result in temperature drift. Temperature drift can be corrected using temperature compensation circuitry, temperature regulation, auto null circuitry, chopping, and isothermal protection (Omega Engineering, 2000). Although a narrow FOV IRT has the advantage of sensing temperature from a homogeneous target when aimed from the appropriate distance, there is the disadvantage that a greater percentage of the total incident radiation received by the detector will be from its own temperature due to internally reflected radiation. This makes narrow FOV sensors more sensitive to changes in their own body temperature (Bugbee et al., 1999).

Currently, there are a limited number of low cost wireless IRT sensors available for retail. An IRT with a narrow FOV has the advantage of viewing a homogeneous sample at a height fixed above the crop canopy under partial canopy coverage, but the inherent problem of temperature output drift due to changes in ambient air temperature have to be overcome. Our objectives for this study were to: (1) develop a low cost, wireless, narrow FOV IRT sensor; and (2) evaluate the accuracy of the output readings under changing ambient temperatures and direct radiation. A brief evaluation of $\mathrm{RF}$ signal strength of the sensor module was performed to indicate the range of communication using whip antennas.

\section{Materials and methods}

\subsection{Sensor specification}

The specifications of the infrared thermometer sensor, MLX90614-BCF (Melexis, Ypres, Belgium) are summarized in Table 1 . This sensor was chosen because of its relatively low cost, narrow FOV (collimated to $10^{\circ}$ by the manufacturer), and capability for non-contact radiometric surface temperature measurements. The sensor package is comprised of a long-wave filter that passes radiation from 5.5 to $14 \mu \mathrm{m}$, a thermopile detector chip and a second unique proprietary signal conditioning chip. The two voltages $\left(V_{s}\right.$ and $\left.V_{o}\right)$ corresponding respectively to: (1) the sensor body temperature $\left(T_{s b}, \mathrm{~K}\right)$; and (2) the object or target temperature $\left(T_{o b j}, \mathrm{~K}\right)$ which is internally compensated by $T_{s b}$. Voltage outputs were converted to temperature readings using the equation, $T_{i}=V_{j} \times 0.02$ in $\mathrm{K}$ and later converted to ${ }^{\circ} \mathrm{C}$, where $T_{i}$ represents either $T_{o b j}$ or $T_{s b}$ and $V_{j}$ is either $V_{o}$ or $V_{s}$. As with any radiometric sensor, the accuracy of this thermometer can be influenced by thermal gradients induced across the sensor package (Melexis Data Sheet, 2009). 
Table 1

Wireless sensor module characteristics.

\begin{tabular}{ll}
\hline Major components & Characteristics \\
\hline Melexis IR detector & $10^{\circ}$ field of view \\
& Wavelength pass $(\mu \mathrm{m}): 5.5-14$ \\
& Resolution/accuracy: $0.02 / \pm 0.5^{\circ} \mathrm{C}$ from 0 to $50^{\circ} \mathrm{C}$ \\
XBee RF Module & Communication protocol $=802.15 .4$ with Zigbee stack \\
& Frequency $=2.4 \mathrm{GHz}$ ISM \\
& Maximum outdoor range $=120 \mathrm{~m}$ \\
& Peak transceiver current $=50 \mathrm{~mA}$ \\
Sensor module & Microprocessor controlled $^{\mathrm{C}}$ \\
& Current draw (sleep $^{\mathrm{a}} /$ transmission $^{\mathrm{c}}$ mode $^{\mathrm{b}}$ ): $: 30 \mu \mathrm{A} / 40 \mathrm{~mA}$ \\
& Cost $^{\mathrm{c}}: \$ 150$
\end{tabular}

a Current draw when the RF module is idle and the microprocessor is awake.

b Current draw when RF module is transmitting to base station.

c Costs are approximate and include IR detector, microprocessor, batteries, and IC components.

We designed electronic circuitry involving a microprocessor to interface the digital output of the Melexis IRT and analog output of the battery pack with a wireless Zigbee RF module (XBee platform, Series 2, Digi International, Minnetonka, Minn.). Sensor data (i.e. $T_{o b j}, T_{s b}$ ), battery voltage, and IRT address (configured with dip switches), were collected and stored at the microprocessor level until sent to the base station lap top computer using RF telemetry. Temperature data were collected every $12 \mathrm{~s}$, averaged and stored every minute. Battery voltage was measured and stored every minute. Transmission frequency, data averaging, and the cyclic period for the "sleep" state of the RF module were programmed through the microprocessor.

The circuit was designed with two voltage regulators, one controlling power exclusively to the Melexis sensor. The average current draw after testing 5 sensor modules was $0.5 \mathrm{~mA}$ when the voltage regulator to the Melexis sensor was powered "off." The average current draw was $10 \mathrm{~mA}$ when the sensor was powered "on." During data transmission, the sensor modules drew an aver- age of $33 \mathrm{~mA}$, which is governed by the design of the XBee series II module. Application code for the sensor module processor was created using CodeVision AVR (V2.04.6, Progressive Resources, LLC, Indianapolis, Ind.), which is a commercial C compiler for Atmel AVR microcontrollers. Data were arranged into the application programming interface (API) format to extend the level of interaction between the sensor nodes and the host application (Fig. 2). Use of the API format allows detection of a complete data string by recognizing the start of the data packet, "7E" (a static value), size of the array to parse (based on the number of bytes in the data string), confirmation that the string was received from a sensor (based on the packet type and address of the sending node), and a means for error checking the data sent by validating the received checksum value.

Each sensor module was powered with 4 AA nickel metal hydride (NiMH) batteries. The batteries were housed in an external white, weather proofed poly vinyl chloride (PVC) housing and recharged with a $3 \mathrm{~W}$ solar panel (model SC3, Sunwize SolCharger, Hudson, Mass.). The combination of off-the-shelf components and circuit design will hereinafter be referred to as a wireless IRT (Fig. 3). Wireless data for the temperature comparisons discussed in this manuscript were collected by an XBee modem connected to a laptop computer using a program running under Visual Studio 2008 Ver. 9.2. (Microsoft Corp., Redmond, Wash.).

\subsection{Temperature comparisons}

To evaluate the accuracy of the wireless IRTs and reduce uncertainties in error measurement, three methods were used: (1) calibration against a variable blackbody; (2) spot temperature comparisons of soil and vegetation samples against a commercial hand-held infrared thermometer; and (3) continuous temperature comparisons over a highly uniform target against an industrial wired IRT in the outdoors.

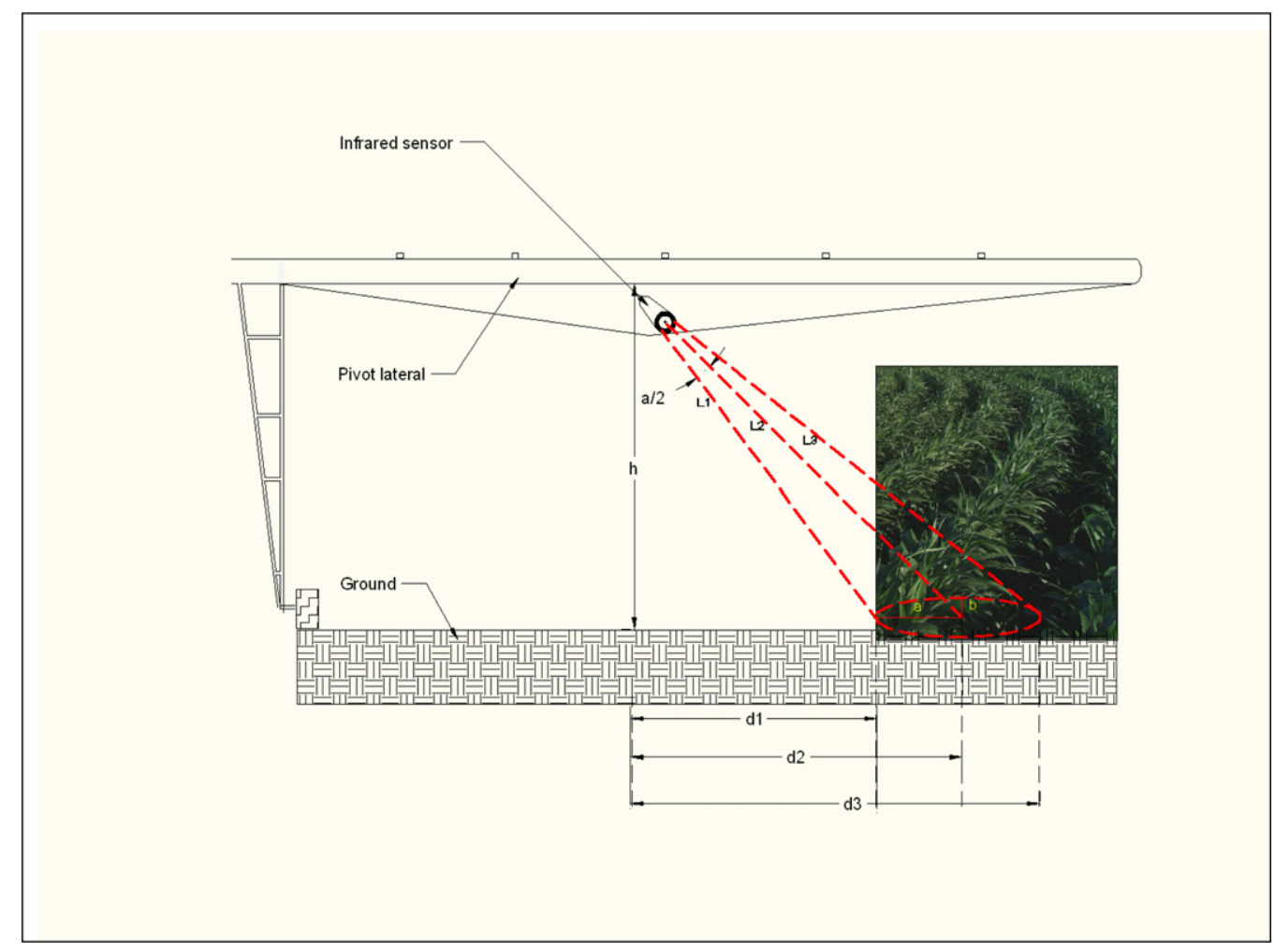

Fig. 1. Graphic showing location of wireless infrared thermometer on pivot lateral and projected field of view (elliptical footprint with axis $a$ and $b$ ). 


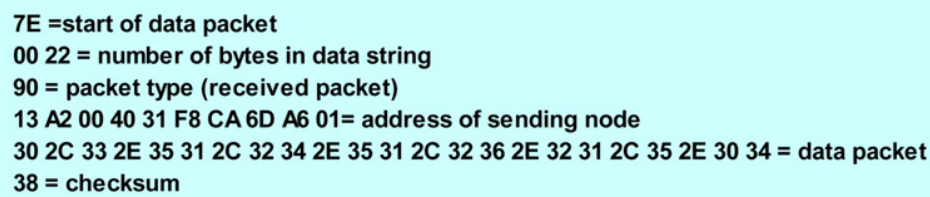

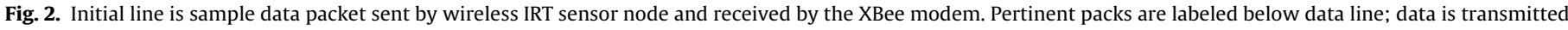
in hexadecimal.

\subsubsection{Sensor vs. blackbody calibrator}

Temperature output instability of the wireless IRTs caused by changes in ambient air temperature was measured by comparing the IRT readings to the known set point temperature of the blackbody in a number of different ambient temperatures. The variable temperature blackbody calibrator (CES100, Electro Optical Industries, Inc., Santa Barbara, Calif.) and the wireless IRTs were placed inside of a $3 \mathrm{~m} \times 3 \mathrm{~m} \times 3 \mathrm{~m}$ temperature control chamber (Environmental Growth Chambers, Inc., Chagrin Falls, Ohio). The chamber and sensor body temperatures were brought to equilibrium, after which batch readings from the sensors were collected. A data logger (Campbell Scientific, model CR 3000, Logan, Utah) was used to automatically control the set point of the blackbody and its chiller unit. A special character was broadcast by the data logger through an XBee modem to the wireless IRT sensor modules when the blackbody had reached a programmed set point. Temperatures, $T_{o b j}$ and $T_{s b}$, and battery voltage measurements were sent via RF telemetry, only after receiving a special character, to a laptop computer located just outside the chamber. The blackbody temperature was automatically stepped from 15 to $55^{\circ} \mathrm{C}$ (the expected range of canopy or soil temperatures during the growing season) in 5-degree increments using an RS-232 communication link between the data logger and blackbody controller. Twenty data samples were collected from each sensor at each blackbody set point for a total of 180 data points per sensor per ambient air temperature. Wireless sensor IRT readings were taken in four different ambient temperatures $\left(15^{\circ} \mathrm{C}, 25^{\circ} \mathrm{C}, 35^{\circ} \mathrm{C}\right.$, and $\left.45^{\circ} \mathrm{C}\right)$. These represented ambient temperature ranges that were expected during a typical summer growing season at Bushland, TX (CPRL Climate Data files, 2010). Although our technique was automated, it was similar to methods performed by Kalma et al. (1988) and Bugbee et al. (1999).

\subsubsection{Wireless IRT vs. AgriTherm hand-held thermometer}

The wireless IRT was evaluated against the AgriTherm II, a portable hand-held infrared thermometer (Everest Interscience Inc., Tucson, Ariz.). The sensor module and AgriTherm II were clamped onto separate masts and placed alongside one another with a nadir view angle over soil and vegetation samples. The soil was $0.25 \mathrm{~m}$ (depth) of dry Pullman Clay Loam spread evenly over a $0.46 \mathrm{~m} \times 0.31 \mathrm{~m}$ pan. Vegetation temperature readings were taken over grain sorghum (Sorghum bicolor (L.) Moench). The AgriTherm II had a zoom lens and pulsating laser; its field of view was adjusted to meet that of the wireless IRT and it emissivity was dialed to 0.98 .

\subsubsection{Wireless IRT vs. wired IRT and thermal imager}

A third comparison involved the use of a wireless IRT and one wired IRT (Exergen model IRT/c.5-T-80F/27C) mounted on masts and positioned above a black rectangular aluminum block target located outdoors. The wired IRT contained a Type-T thermocouple (copper-constantan) detector with specifications similar to the thermopile detector in the wireless IRT. The two sensors had the same FOV, were filtered to select spectral responses in the 8 to $14 \mu \mathrm{m}$ range, and measured at comparable levels of accuracy near room temperature. The aluminum block target over which both type of sensors were positioned had dimensions of $81.3 \mathrm{~cm} \times 38.1 \mathrm{~cm} \times 7.6 \mathrm{~cm}$ (tall), and a measured emissivity value of 0.99 . The method to measure emissivity is described in (Kaplan, 2007). Temperature measurements between the wired and wireless IRTs were made over a 24-h period. Data from the wireless IRTs were collected by a lap top computer located in a remote building and time stamped by the acquisition program. The wired IRT did not require excitation, rather the voltage differences generated between the dissimilar wire types were amplified, collected, and
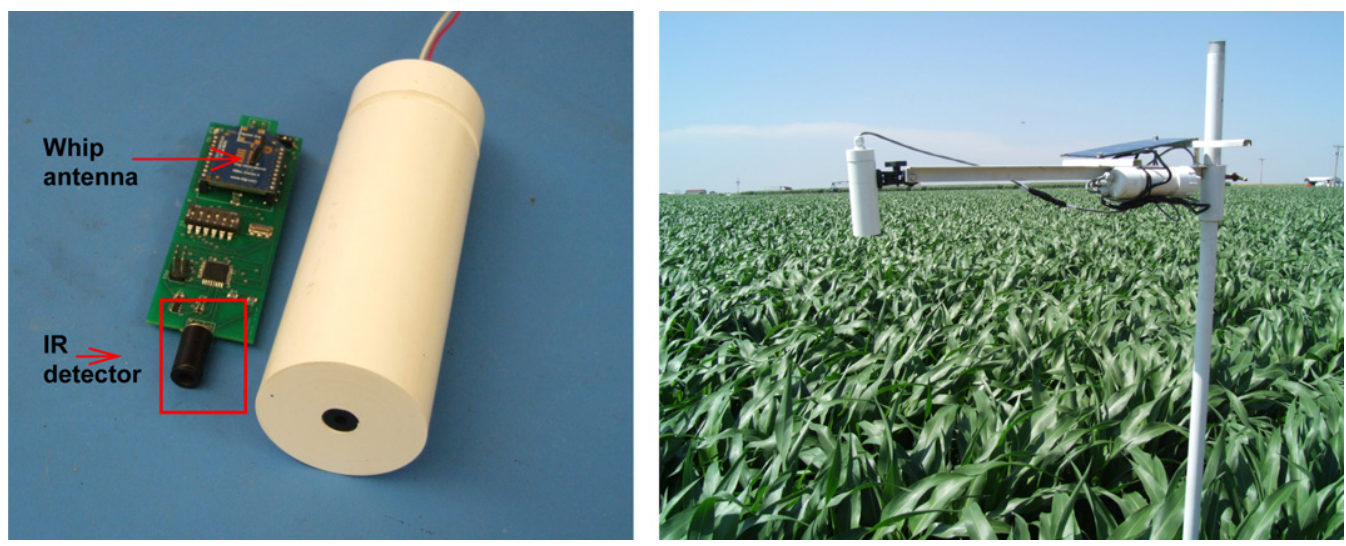

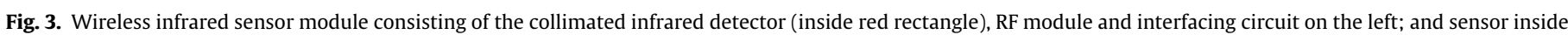
of the white PVC housing on the right. 
time stamped by a data logger (model 21X, Campbell Scientific, Logan, Utah). The clocks for the computer and data logger were synchronized prior to data collection for paired analysis.

The temperature of the aluminum block was extracted from digital images taken periodically with a FLIR thermal imager (model SC2000 and ThermaCAM Researcher Pro 2.8 software, FLIR Systems, Billerica, Mass.) at a nadir view angle on DOYs 89, 90 and 91, 2010.

\subsubsection{Response to temperature transients with and without a heat sink}

Thermal mass requirements for sensor body temperature stabilization were investigated with the detector exposed to heating caused by direct radiation while: (1) embedded in an aluminum socket and the detector and circuit interface board enclosed inside of a white polyvinyl chloride (PVC) plastic sleeve; and (2) with the detector and circuit interface board enclosed only in the plastic sleeve. Transient incident radiation trials were used to simulate rapid changes in radiation on the sensor, which would affect sensor body temperature. Such changes may occur, for example, when the sun is intermittently blocked by cloud cover. All trials were performed in the controlled temperature room. During each trial, the sensor module was aimed at the blackbody target with a constant target temperature, $T_{B B}\left({ }^{\circ} \mathrm{C}\right)$. Incident radiation was increased and decreased by turning on and off a halogen lamp aimed at the sensor module. A shield isolated the blackbody from the radiation. The blackbody target was varied in 5-degree increments as described previously, and the testing was repeated in ambient temperatures of $20^{\circ} \mathrm{C}, 25^{\circ} \mathrm{C}, 30^{\circ} \mathrm{C}$, and $40^{\circ} \mathrm{C}$.

Temperature readings of wireless IRTs were compared with those from the blackbody, wired IRT, hand-held IRT and thermal imager using linear regression analysis. Statistics including root mean square error (RMSE):

$$
\sqrt{\frac{\sum_{i=1}^{n}\left(O_{i}-P_{i}\right)^{2}}{(n-1)}}
$$

where $O_{i}$ is the $i$ th measurement, $P_{i}$ is the $i$ th predicted value, $n$ is the total number of data pairs. Root mean square error is the standard deviation of measurements and characterizes the reliability of measurements (Taylor, 1997). The mean absolute error (MAE):

$$
\frac{\sum_{i=1}^{n}\left|O_{i}-P_{i}\right|}{n}
$$

is a statistic that indicates the average magnitude of error between the observed and predicted (forecasted) measurement. Its use is preferred for small or limited data sets. Finally the mean biased error (MBE) described by Mandel (1964):

$=\sum_{i=1}^{n} \frac{\left(\mu-R_{i}\right)}{n}$

reports the inaccuracy or difference in the sample mean or expected value $(\mu)$ and the reference value $\left(R_{i}\right)$ for each measurement. This equation was used to identify systematic or non-random errors. Standard deviation from the mean or the non-negative square root of the variance was used to detect variability about the mean for measurements made by a single instrument.

Finally, since it is not possible to measure the true temperature of an object especially in the outdoors, the standard error of the predicted $y$-value for each $x$ in the regression was used to measure the amount of error in the prediction of readings from an industrial wired IRT (the predicted values) for an individual reading from a
Table 2

\begin{tabular}{|c|c|c|c|c|c|}
\hline \multirow[t]{3}{*}{ Sensor No. } & \multicolumn{3}{|c|}{ Untreated data } & \multicolumn{2}{|c|}{ Linear calibration applied } \\
\hline & RMSE & MAE & MBE & RMSE & MAE \\
\hline & ${ }^{\circ} \mathrm{C}$ & & & & \\
\hline 11 & 0.36 & 0.68 & 0.67 & 0.23 & 0.40 \\
\hline 12 & 0.28 & 0.52 & 0.22 & 0.29 & 0.55 \\
\hline 13 & 0.16 & 0.25 & 0.10 & 0.16 & 0.24 \\
\hline 14 & 0.16 & 0.25 & -0.20 & 0.13 & 0.19 \\
\hline 15 & 0.59 & 1.11 & -1.12 & 0.25 & 0.29 \\
\hline 16 & 0.79 & 1.52 & 1.52 & 0.30 & 0.47 \\
\hline 17 & 0.24 & 0.25 & 0.23 & 0.20 & 0.15 \\
\hline 18 & 0.15 & 0.27 & 0.22 & 0.15 & 0.23 \\
\hline 19 & 0.25 & 0.42 & 0.38 & 0.22 & 0.36 \\
\hline 20 & 0.13 & 0.19 & -0.08 & 0.12 & 0.17 \\
\hline 21 & 0.16 & 0.26 & 0.25 & 0.11 & 0.13 \\
\hline 22 & 0.37 & 0.69 & 0.66 & 0.20 & 0.33 \\
\hline 23 & 0.32 & 0.53 & 0.12 & 0.31 & 0.53 \\
\hline 24 & 0.27 & 0.50 & 0.12 & 0.27 & 0.50 \\
\hline 25 & 0.21 & 0.37 & 0.12 & 0.23 & 0.39 \\
\hline 26 & 0.20 & 0.34 & 0.26 & 0.26 & 0.32 \\
\hline 27 & 0.33 & 0.63 & 0.61 & 0.12 & 0.17 \\
\hline 28 & 0.37 & 0.68 & 0.70 & 0.11 & 0.13 \\
\hline 29 & 0.13 & 0.69 & 0.17 & 0.10 & 0.13 \\
\hline 30 & 0.17 & 0.27 & 0.76 & 0.10 & 0.12 \\
\hline 31 & 0.14 & 0.19 & 0.05 & 0.12 & 0.16 \\
\hline 32 & 0.20 & 0.36 & 0.33 & 0.17 & 0.28 \\
\hline 33 & 0.14 & 0.18 & 0.04 & 0.13 & 0.16 \\
\hline 34 & 0.21 & 0.37 & 0.36 & 0.12 & 0.17 \\
\hline
\end{tabular}

IRT readings compared against blackbody calibrator.

wireless IRT (the $x$ value). The equation for the standard error is:

$\sqrt{\frac{1}{(n-2)}}\left[\sum(y-\bar{y})^{2}-\frac{\left[\sum(x-\bar{x})(y-\bar{y})\right]^{2}}{\sum(x-\bar{x})^{2}}\right]$

where $y$ and $\bar{y}, x$ and $\bar{x}$ are the samples and mean for the wired IRT and wireless IRTs, respectively, and $n$ is the number of paired samples. The standard error is a measure of the amount of error in the prediction of $y$ for an individual $x$, but for our purposes, it was used to evaluate how well measurements from the two instruments agreed.

\subsection{RF communications}

RF communications were tested by measuring the received signal strength indicator (RSSI, measured in $\mathrm{dB}$ ) for a sensor node at each of five separation distances $(7.5 \mathrm{~m}, 15 \mathrm{~m}, 30 \mathrm{~m}, 60 \mathrm{~m}$, and $120 \mathrm{~m}$ ). Signal strength is best measured by sending a number of packets and calculating the percent of packets received (AndradeSanchez et al., 2007; Hebel et al., 2007; O'Shaughnessy and Evett, 2010). At each distance, 25 packets of data were broadcast to the sensor node and the percent of successful receptions was recorded. Both the sensor node and the modem were enclosed inside of a PVC housing with an XBee series 2 (S2) module and whip antenna, Fig. 3. The signal strength of the antenna was equal to $1.5 \mathrm{dBi}$ (gain of the antenna referenced to an isotropic source), and positioned at $1.5 \mathrm{~m}$ above the ground during testing. This is the height at which we typically place our IRTs relative to crop canopy height.

\section{Results and discussion}

\subsection{IRT vs. blackbody calibrator}

The RMSE values calculated between the wireless IRT sensor readings and blackbody target temperatures ranged from 0.10 to $0.79^{\circ} \mathrm{C}$ for measurements made in all ambient temperatures (Table 2). Compared to the standard accuracy of the Melexis, reported to be $\pm 0.5^{\circ} \mathrm{C}$, only two of the tested sensors (\#15 and \#16) 

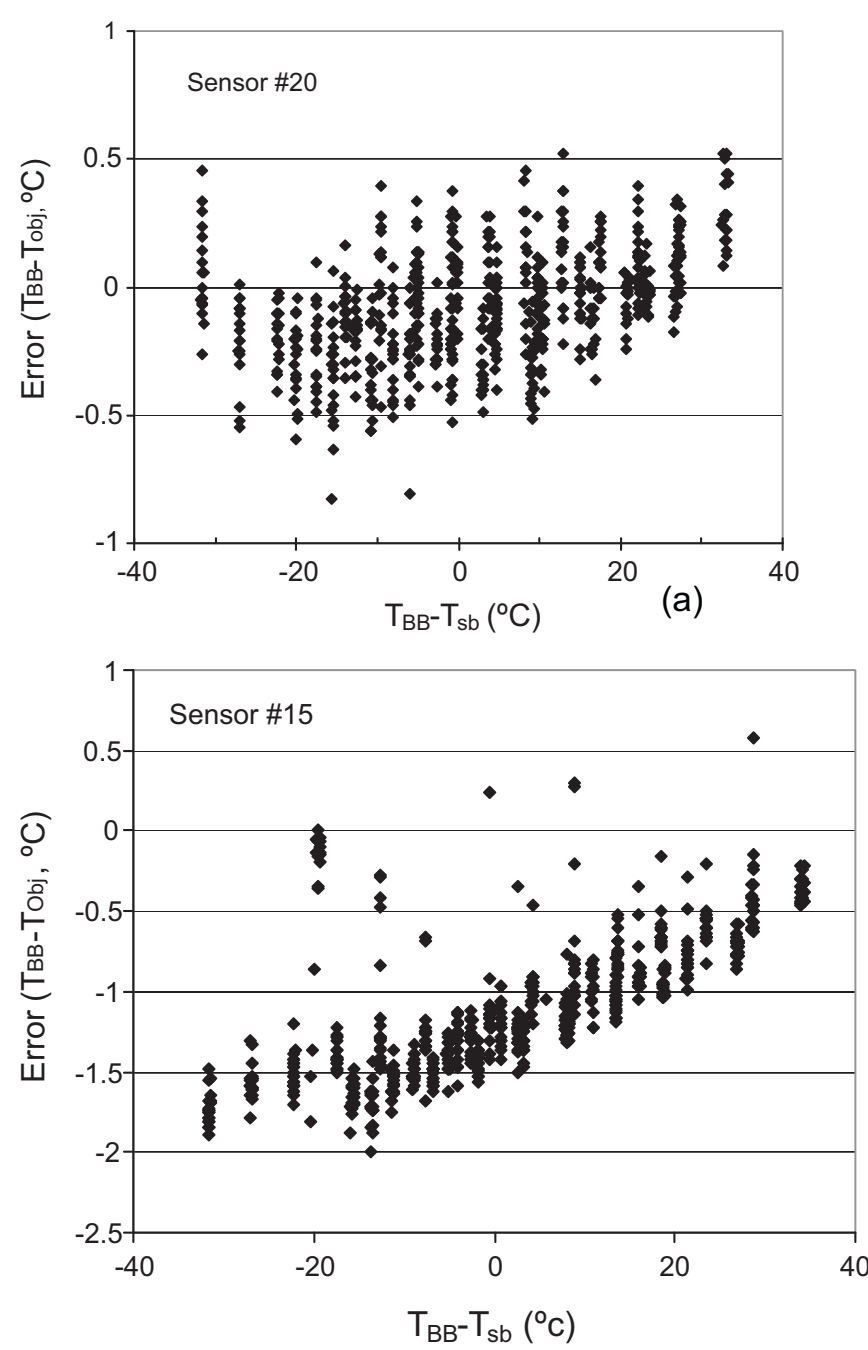

Fig. 4. Comparison of IRT performance between two wireless IRTs showing residual errors or differences in blackbody target $\left(T_{B B}\right)$ and wireless IRT $\left(T_{o b j}\right)$ temperature readings across a range of $T_{B B}$ and sensor body $\left(T_{s b}\right)$ temperature differences from $-40^{\circ} \mathrm{C}$ to $+40^{\circ} \mathrm{C}$ for: (a) sensor $\# 20$ having a RMSE $=0.16^{\circ} \mathrm{C}$ and $\mathrm{MBE}=-0.08^{\circ} \mathrm{C}$; and (b) sensor \#15 having a RMSE $=0.59^{\circ} \mathrm{C}$ and $\mathrm{MBE}=-1.12^{\circ} \mathrm{C}$.

had a RMSE above $0.5^{\circ} \mathrm{C}$. The significance of a low RMSE value indicates a high level of accuracy; $92 \%$ of the tested sensors provided accurate temperature measurements against the variable blackbody calibrator in changing ambient temperatures. The MAE ranged from 0.12 to $1.52{ }^{\circ} \mathrm{C}$ for all sensors compared against the blackbody calibrator. Again the highest error values were contributed by sensors \#15 and \#16. This error statistic indicates that these two sensors can output an error in temperature reading that is 2-3 times greater in magnitude than the other sensors. The MBE for twenty sensors ranged from -0.5 to $0.5^{\circ} \mathrm{C}$. These values being relatively close to $0.0^{\circ} \mathrm{C}$ and within the specifications provided by the manufacturer, imply limited bias. On the other hand, the relatively high MBE levels for sensors \#15 $\left(-1.12^{\circ} \mathrm{C}\right)$ and \#16 $\left(1.52^{\circ} \mathrm{C}\right)$ suggest systematic errors. Review of the data for sensor \#15 showed that the IRT readings were consistently higher than the blackbody calibrator, while data for sensor \#16 indicated that its readings were consistently lower than the target. Fig. 4a provides a graphic of both the accuracy [range of residual errors, i.e. differences in temperature between the target $\left(T_{B B}\right)$ and the IRT reading $\left(T_{o b j}\right)$ ] shown along the ' $y$-axis' for sensor \#20. The error residuals generally ranged from -0.6 to $0.6^{\circ} \mathrm{C}$ regardless of the temperature difference between $T_{B B}$ and $T_{S b}$ shown along the ' $x$-axis'; this indicated that the sensor output remained relatively accurate when sensor body

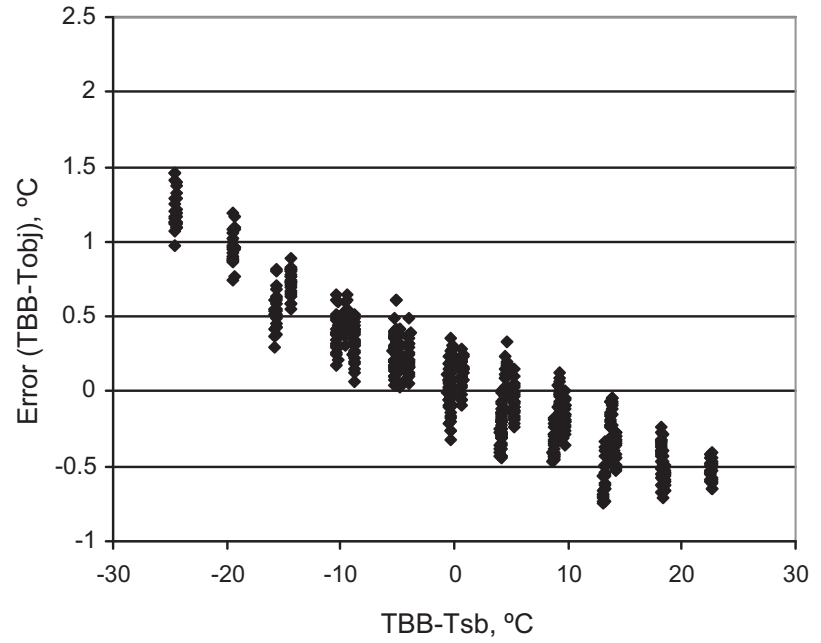

Fig. 5. Error (difference between target blackbody temperature $\left(T_{B B}\right)$ and IRT temperature reading $\left(T_{o b j}\right)$ plotted against the difference between $T_{B B}$ and sensor body temperature $\left(T_{s b}\right)$ for sensor module housed in an aluminum block and inserted in a white PVC sleeve. Comparisons were made in ambient temperatures of $20^{\circ} \mathrm{C}, 25^{\circ} \mathrm{C}$ $30^{\circ} \mathrm{C}$, and $40^{\circ} \mathrm{C}$.

temperature changed. In contrast, the errors in readings from sensor \#15 were consistently greater than $0.5^{\circ} \mathrm{C}$ whenever the $T_{s b}$ was equal to or greater than $T_{B B}$; and the majority of the errors were negative, Fig. 4b. The negative bias and sources of error may stem from any number of issues including imprecision of the temperature compensating chip or problems associated with the interface board. Probable examples include electronic noise caused by imperfections in the interface circuit that we constructed, heat build-up during the operation of the sensor or from the heat given off by the blackbody calibrator, or misalignment against the blackbody calibrator target when the sensors were tested in batches.

The simple linear regression equation was used to calibrate each sensor and did not include sensor body temperature as a variable to adjust the sensor output reading:

$\Upsilon=m_{i} T_{o b j}+b_{i}$

where $Y$ was the measured value of the blackbody target, and $m_{i}$ and $b_{i}$ are the customized slope and intercept from the regression. This calibration equation reduced the RMSE value in most cases, Table 2. The use of simple linear equations is different from those used by Bugbee et al. (1999) where in their studies, the slope and intercept were parameterized as a function of $T_{s b}$ using higher order polynomials. Applying the correction techniques of Bugbee et al. (1999) to the wireless IRT sensors only slightly increased the RMSE values (data not shown) for our sensors.

\subsection{Response to temperature transients with and without a heat sink}

When direct radiation was applied to the detector embedded inside of an aluminum socket and white PVC sleeve while the blackbody temperature was varied (in different ambient temperatures), the detector responded by decreasing voltage output $\left(V_{o}\right)$ for the $T_{o b j}$ readings when $T_{s b}$ was greater than $T_{B B}$, and increasing $V_{o}$ when $T_{s b}$ was less than $T_{B B}$ (Fig. 5). In other words, the detector under compensated when the sensor body temperature was greater than the blackbody temperature $\left(T_{B B}\right)$ and overcompensated when the $T_{B B}$ was more than $10^{\circ} \mathrm{C}$ greater than $T_{S b}$. With the detector embedded in the heat sink, the maximum error ranged from -0.8 to $1.5^{\circ} \mathrm{C}$ which was greater than the manufacturer's specifications of $0.5^{\circ} \mathrm{C}$ under isothermal conditions. The MBE was not significantly different when the detector was embedded in the aluminum housing 
Table 3

Average and mean difference of temperatures of 10 samples from a wireless sensor module and handheld IRT taken at different times of the day. SD represents one standard deviation from the mean temperature. Mean absolute error (MAE) shows the magnitude of the error between instruments.

\begin{tabular}{|c|c|c|c|}
\hline \multirow[t]{3}{*}{ Sample type (time) } & \multicolumn{3}{|l|}{${ }^{\circ} \mathrm{C}$} \\
\hline & \multicolumn{3}{|c|}{ Object temperature readings $\pm S D$} \\
\hline & Sensor module & AgriTherm II & MAE \\
\hline Soil (09:00 h) & $17.10 \pm 0.04$ & $16.99 \pm 0.03$ & 0.11 \\
\hline Vegetation (09:05 h) & $15.18 \pm 0.03$ & $15.10 \pm 0.00$ & 0.17 \\
\hline Vegetation (13:15 h) & $29.01 \pm 0.03$ & $28.22 \pm 0.04$ & 0.84 \\
\hline Soil $(13: 27 \mathrm{~h})$ & $29.32 \pm 0.03$ & $28.67 \pm 0.08$ & 0.70 \\
\hline Soil (14:55 h) & $30.48 \pm 0.09$ & $29.68 \pm 0.04$ & 0.85 \\
\hline Vegetation $(15: 00 \mathrm{~h})$ & $27.37 \pm 0.01$ & $26.82 \pm 0.08$ & 0.60 \\
\hline
\end{tabular}

$\left(0.08^{\circ} \mathrm{C}\right)$ compared to $0.09^{\circ} \mathrm{C}$ when the detector was located only in the PVC sleeve.

Further stability testing was performed by investigating the effects of direct radiation over a short period of time and under a constant ambient temperature near $20^{\circ} \mathrm{C}$ with the detector embedded inside of a heat sink and the detector and interface circuit board inside of the PVC housing. There was no significant difference in $T_{o b j}$ readings (Fig. 6a). While embedded inside of the heat sink, the sensor body temperature rose by $4{ }^{\circ} \mathrm{C}$ after approximately $30 \mathrm{~min}$ (time frame corresponding to sampling between 27 and 107 on the horizontal scale) of direct heating. This test was repeated four times and the average RMSE between $T_{o b j}$ and the blackbody target temperature was $0.04{ }^{\circ} \mathrm{C}$, while the MAE was $0.33^{\circ} \mathrm{C}$.

With the detector removed from the heat sink but enclosed inside the PVC housing with the interfacing circuit board, direct heat was again applied, $T_{s b}$ rapidly increased over a short period of time, however, $T_{o b j}$ readings remained very near to $T_{B B}$ (Fig. $6 \mathrm{~b}$ ). The RMSE between $T_{o b j}$ and $T_{B B}$ was $0.05^{\circ} \mathrm{C}$ and the MAE was $0.50^{\circ} \mathrm{C}$. The stability of the object temperature reading under the influence of direct radiation and no heat sink demonstrated that the compensation feature of the sensor functioned well to maintain accurate temperature readings relative to $T_{B B}$ and that the heat sink did not provide additional benefits. It should be noted that in both cases, the $T_{o b j}$ was greater than $T_{B B}$ however the change in $T_{o b j}$ was not significant over time and can be corrected using an offset.

Realizing that it is not possible to measure the true temperature of an object, the next two experiments were completed to determine the differences between IRT measurements and those from accepted instruments, i.e. readings from a commercial hand-held IRT and an industrial IRT. The differences in measurements were not to be considered errors, but rather were used to characterize the magnitude of variation among and between IRT instruments.

\subsection{Hand-held and wireless comparisons}

Multiple readings taken over vegetation and soil at different times of the day allowed us to evaluate the precision of the wireless and hand-held IRT. The standard deviation of measurements shown for each instrument over each sample type and time period ranged from 0.00 to $0.08{ }^{\circ} \mathrm{C}$ for the Agritherm II and from 0.07 to $0.15^{\circ} \mathrm{C}$ for the wireless IRT. This indicates that the variability of temperature readings for each instrument was small and that the Agritherm II instrument displayed less variability. The MAE represents the magnitude of difference between the instruments over the different samples and time periods. A difference of less than $1{ }^{\circ} \mathrm{C}$ indicates that the measurements were in good agreement, Table 3.

\subsection{Wired and wireless comparisons}

This study resulted in paired temperature measurements between a wireless and industrial wired IRT over a two-day period in ambient temperatures ranging from 10 to $45^{\circ} \mathrm{C}$. The standard error of the predicted reading (the wired IRT), for each reading from the wireless IRT was $0.32^{\circ} \mathrm{C}(07: 55-15: 42 \mathrm{~h})$ and $0.26^{\circ} \mathrm{C}$ (16:32-02:30 h) on DOY 89-90, and $0.43^{\circ} \mathrm{C}(07: 55-15: 42 \mathrm{~h})$ and $0.40{ }^{\circ} \mathrm{C}(16: 32-02: 30 \mathrm{~h})$ when viewing the aluminum block target on DOY 90-91. The relatively low standard error values demonstrate that the wireless IRT readings compared well with those from the commercial wired IRT, Fig. 7.

Three thermal images were taken consecutively at 1-min intervals at each of four different start times throughout the day. Measurements from both IRTs evaluated against the extracted temperatures from the FLIR images were in good agreement. Evaluating the accuracy of both IRTs against data from the FLIR camera (the predicted value) using the RMSE statistic indicated that the wireless IRT $\left(\mathrm{RMSE}=0.55^{\circ} \mathrm{C}\right.$ ) was closer in value to readings from the thermal images than the wired instrument $\left(\mathrm{RMSE}=1.10^{\circ} \mathrm{C}\right.$ ), Table 4 . As the ambient air temperature increased, the output of the wired IRT was consistently higher than that of the wireless IRT and data extracted from the FLIR image. The temperature drift of the wired IRT was indicative of this instrument's sensitivity to changes in ambient air temperature and inability to compensate output readings quickly. In general, it is difficult to make concise paired measurements from multiple IRTs even after aligning the sensors side-by-side in an outdoor environment due to the numbers of variables impacting thermal measurements. The aluminum block was used to minimize target variations and improve comparisons between the wired and wireless IRT against a large isothermal target of relatively uniform emissivity (in the outdoors).

\subsection{Radio frequency communication}

Radio communications for three wireless sensors at five different distances from an XBee coordinator, indicated an average peak RSSI of $90 \%$ (of packets received), at an interval distance of $15 \mathrm{~m}$, Fig. 8. The RSSI declined as the distance between the sensors and coordinator increased. There was a consistent dip in signal strength at the $30 \mathrm{~m}$ interval for each sensor tested. The reduced communication range at $30 \mathrm{~m}$ could not be accounted for with the testing that we performed, since the calculated radius of the widest point of the Fresnel zone at a distance of $30 \mathrm{~m}$ between antennas was $0.94 \mathrm{~m}$, the height of the sensors was $1.5 \mathrm{~m}$, and there was no object obstructing the line of the sight between the sensor node and coordinator during the testing. However, the significant decrease in range at the $120 \mathrm{~m}$ interval was expected due the power limitations of the RF module. It should also be noted that enclosure of the RF antenna inside the PVC housing attenuates the signal strength has reported by Darr and Zhao (2008) and O'Shaughnessy and Evett (2010).

\section{Discussion}

Assessing uncertainties in the output readings of these prototype wireless IRTs was important because accurate measurements using these instruments are required in research applications. Although it was impossible to obtain "true" object temperature readings, a number of comparisons were used against instruments with very narrow specifications, and with instruments that have accepted temperature readings such as the commercial hand-held IRT and the industrial wired IRT.

Output readings from the wireless IRTs may have been affected by imperfections in the interface board. Flaws may be detected by measuring the resistance of each component without power 

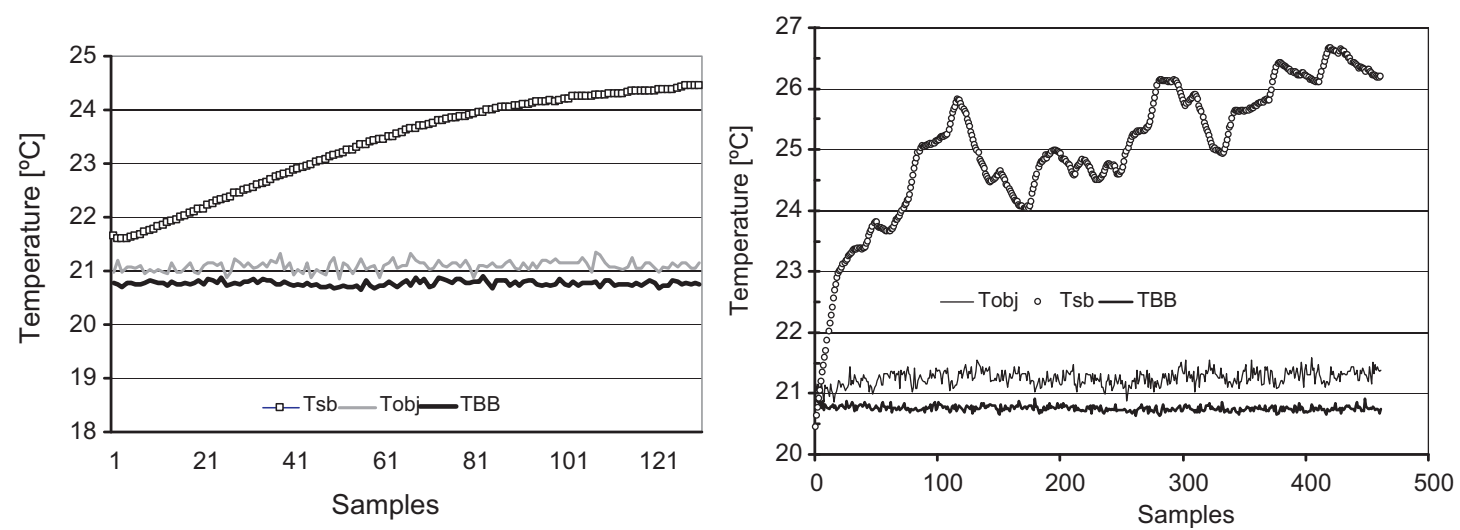

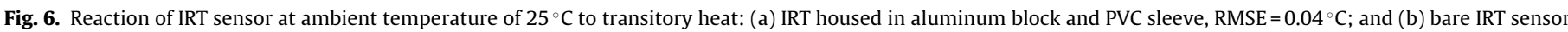
exposed directly to heat, RMSE $=0.05^{\circ} \mathrm{C}$.

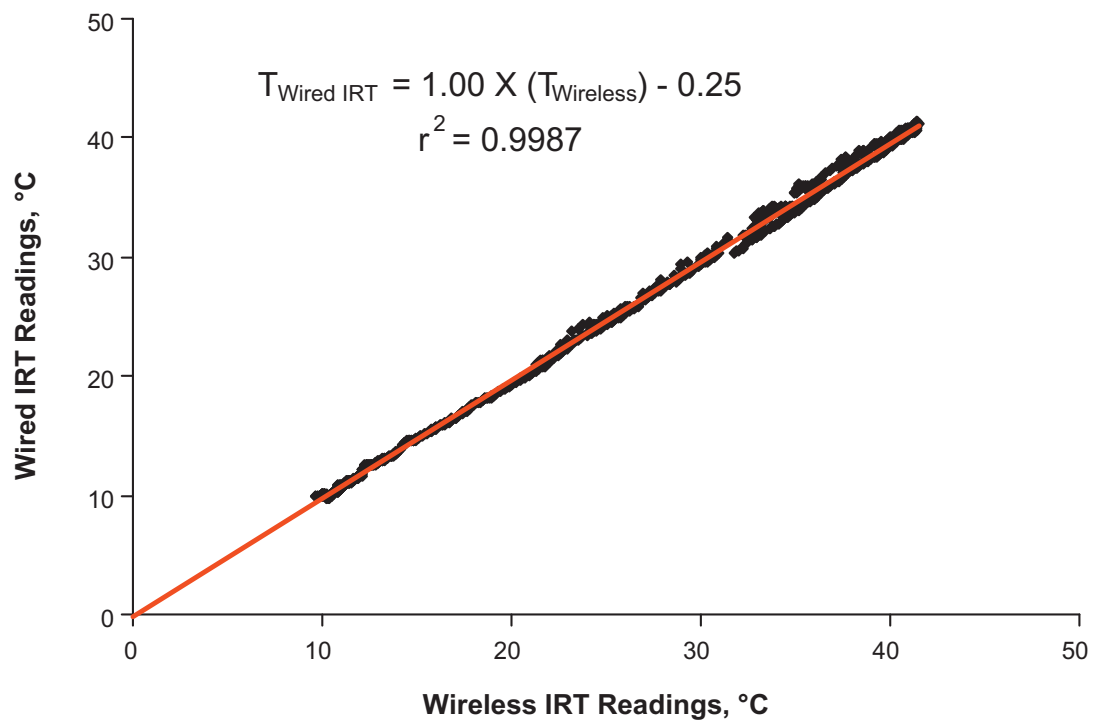

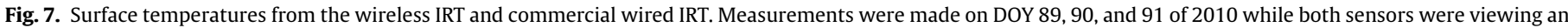
aluminum block.

to the board and then measuring voltages at specific points and comparing the readings to expected values when the board is powered. If excessive heat build-up occurs during the transmission of data (period of highest current draw) and if it impacts the temperature of the IR detector, then it may be possible to limit the heat build-up by troubleshooting for imperfections that are the cause of high impedance, increasing the size of the power traces on the interface board, reviewing the dissipation and efficiency factors of various board components and replacing them with better performing parts, and limiting the length of transmission periods.
Overall, the low RMSE values calculated for the majority of sensors indicated that the detector's temperature compensation algorithm worked well. If built-in temperature compensation is not adequate, then the methods of calibration provided by Kalma et al. (1988) may need to be applied.

It should be noted that the communication evaluation of the wireless sensors was very limited in scope, but the communication range of these IRTs can be improved by substituting a RF module with an external dipole antenna (greater gain) or by increasing the size of the antenna at the coordinator when established within a

Table 4

Comparative averaged radiometric surface temperature readings over a black aluminum block from a wired and wireless IRT and a thermal imager.

\begin{tabular}{|c|c|c|c|c|c|}
\hline Start Time & Wired IRT $\left({ }^{\circ} \mathrm{C}\right)$ & Wireless IRT $\left({ }^{\circ} \mathrm{C}\right)$ & $\operatorname{FLIR}\left({ }^{\circ} \mathrm{C}\right)$ & RMSE $\left({ }^{\circ} \mathrm{C}\right)$ (wired vs. FLIR) & RMSE $\left({ }^{\circ} \mathrm{C}\right)$ (wireless vs. FLIR) \\
\hline 1130 & 24.95 & 24.12 & 24.3 & 0.69 & 0.34 \\
\hline 1131 & 25.03 & 24.3 & 24.4 & & \\
\hline 1132 & 24.87 & 24.07 & 24.5 & & \\
\hline 1158 & 25.6 & 28.55 & 28.3 & 0.81 & 0.66 \\
\hline 1159 & 25.77 & 28.98 & 28.3 & & \\
\hline 1200 & 26.18 & 28.99 & 28.4 & & \\
\hline 1309 & 34.23 & 33.83 & 33.1 & 1.11 & 0.71 \\
\hline 1310 & 34.12 & 33.66 & 33.4 & & \\
\hline 1311 & 33.73 & 33.54 & 32.9 & & \\
\hline 1537 & 39.47 & 38.47 & 37.9 & 1.65 & 0.50 \\
\hline 1538 & 39.57 & 38.35 & 38.3 & & \\
\hline 1539 & 39.27 & 38.5 & 38.1 & & \\
\hline
\end{tabular}




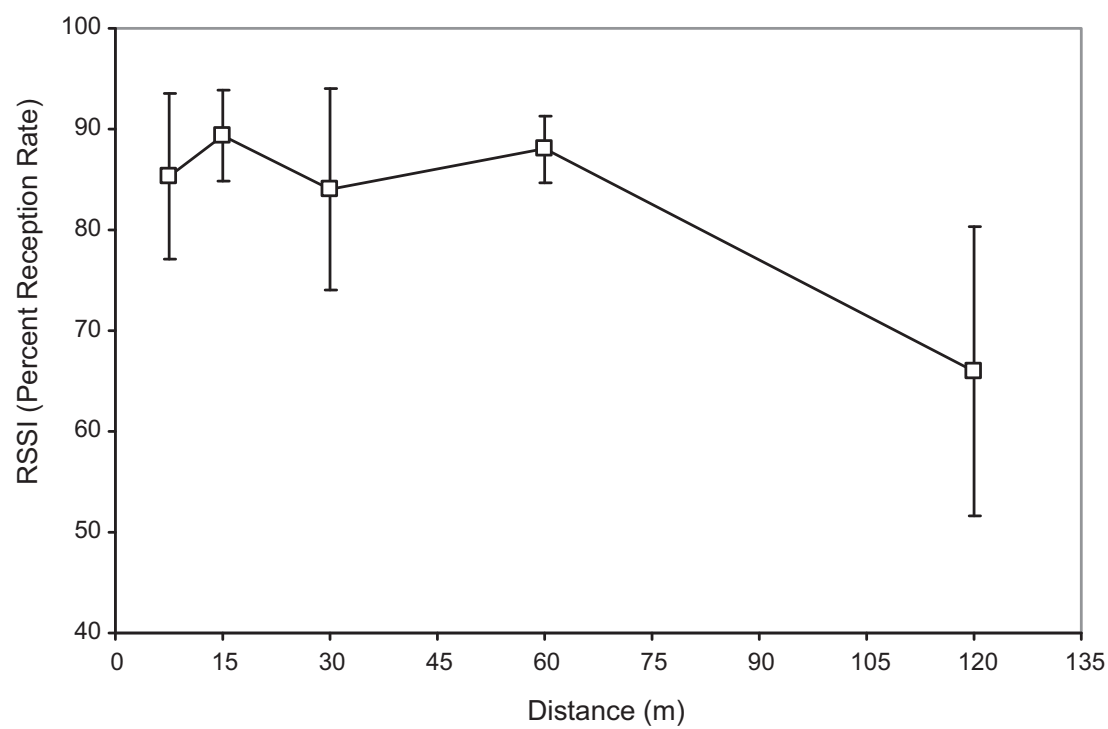

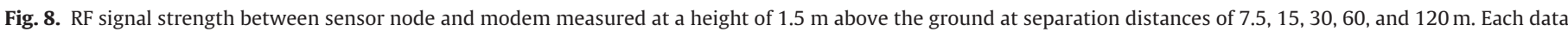
point represents 5 samples, and the vertical bars represent 1 standard deviation.

wireless sensor network. Additionally when working with meshtype wireless sensor networks, the number of sensors in a network and the placement of repeaters can also impact the reliability and range of data communication.

\section{Conclusions}

This study evaluated the performance of prototype narrow FOV wireless IRTs against a blackbody calibrator and measurements from hand-held and wired infrared thermometers. An increase in the availability of narrow FOV wireless IRTs could alleviate challenges for agricultural researchers who are utilizing infrared thermometry to effectively quantify crop water stress and provide instrumentation useful to precision agricultural applications. Surface temperature readings from the wireless IRTs compared well in indoor and outdoor environments, and over soil and vegetation samples. Low RMSE and MAE values indicate that the manufacturer's applied signal conditioning method mitigated the issue of temperature drift under the tested conditions. There was no apparent advantage to using the aluminum socket for purposes of sensor body temperature stabilization which is important in designing suitable and economical housing. Although accuracy is not easy to obtain in outdoor conditions, the comparisons reported by this manuscript indicated that the readings from these prototypes will provide adequate monitoring of crop canopy temperature when positioned on a pivot lateral. However, because PVC housing does not completely isolate the IRT element from changes in ambient air temperature or heating that may be produced by the electronic components on the circuit board, additional testing of the wireless sensor modules in an outdoor setting is necessary to investigate the consistency and accuracy of long term object temperature readings, especially when the difference between sensor body and object temperature is greater than $10^{\circ} \mathrm{C}$.

\section{Acknowledgements}

The authors appreciate the dedicated work performed by Chad Ford and Luke Britten, Agricultural Science Technicians, USDA-ARS, Bushland, TX, and funding for this project from the Ogallala Aquifer Program, a consortium between USDA-Agricultural Research Service, Kansas State University, Texas AgriLife Research,
Texas AgriLife Extension Service, Texas Tech University, and West Texas A\&M University.

\section{References}

Ajayi, A.E., Olufayo, A.A., 2004. Evaluation of two temperature stress indices to estimate grain sorghum yield and evapotranspiration. Agron. J. 96, 1282-1287.

Andrade-Sanchez, P., Pierce, F.J., Elliott, T.V. Performance Assessment of Wireless Sensor Networks in Agricultural Settings. Paper No. 073076. ASABE, St. Joseph, Mich., 2007.

Baker, J.M., Norman, J.M., Kano, A., 2001. A new approach to infrared thermometry. Agric. Forest Meteor. 108, 281-292.

Brewster, M.Q., 1992. Thermal Radiative Transfer and Properties. John Wiley \& Sons, New York.

Bugbee, B., Droter, M., Monje, O., Tanner, B., 1999. Evaluation and modification of commercial infra-red transducers for leaf temperature measurement. Adv. Space Res. 22, 1425-1434.

Colaizzi, P.D., Barnes, E.M., Clarke, T.R., Choi, C.Y., Waller, P.M., 2003. Estimating soil moisture under low-frequency surface irrigation using Crop Water Stress Index. J. Irrig. Drain. Eng. 129, 27-35.

CPRL (Conservation and Production Research Laboratory). Climate Data Files, 2010. Available from: http://www.cprl.ars.usda.gov/climatedatafiles.html.

Darr, M.J., Zhao, L. A Wireless Data Acquisition System for Monitoring Temperature Variations in Swine Barns. Paper No. 987-994. ASABE, St. Joseph, Mich., 2008.

Hebel, M.A., Tate, R.F., Watson, D.G. Results of Wireless Sensor Network Transceiver Testing for Agricultural Applications. Paper No. 073077. ASABE, St. Joseph, Mich., 2007.

IEEE802.15.4. Specific requirements Part 15.4: Wireless Medium Access control (MAC) and Physical Layer (PHY) Specifications for Low-Rate Wireless Personal Area Networks (WPANs). Amendment 1: Add Alternate PHYs. IEEE, Inc., 3 Park Avenue, New York, NY, 2007. http://standards.ieee.org/getieee802/802.15.html (accessed 9.15.2008).

Irmak, S., Haman, D.Z., Bastug, R., 2000. Determination of crop water stress index for irrigation timing and yield estimation of corn. Agron. J. 92, 1221-1227.

Jackson, R.D., Idso, S.B., Reginato, R.J., Pinter, P.J., 1981. Crop canopy temperature as a crop water stress indicator. Water Resour. Res. 17, 1133-1138.

Jones, H., 2004. Irrigation scheduling: advantages and pitfalls of plant-based methods. J. Exp. Bot. 55, 2427-2436.

Kalma, J.D., Alksnis, H., Laughlin, G.P., 1988. Calibrations of small infra-red surface temperature transducers. Agric. Forest Meteor. 43, 83-98.

Kalma, J.D., Jupp, D.L.B., 1990. Estimating evaporation from pasture using infrared thermometry: evaluation of a one-layer resistance model. Agric. Forest Metero. 51, 223-246.

Kaplan, H., 2007. Practical Applications of Infrared Thermal Sensing and Imaging Equipment, 3rd ed. Society of Photo-Optical Instrumentation Engineers, Bellingham, Wash.

Kustas, W.P., Norman, J.M., 1997. A two-source approach for estimating turbulent fluxes using multiple angle thermal infrared observations. Water Resour. Res. $33,1495-1508$

Mahan, J.R., Yeater, K.M., 2008. Agricultural applications of a low-cost infrared thermometer. Comput. Electron. Agric. 64, 262-267. 
Mahan, J.R., Conaty, W., Neilson, J., Payton, P., Cox, S.B., 2010. Field performance in agricultural settings of a wireless temperature monitoring system based on a low-cost infrared sensor. Comput. Electron. Agric. 71, 176-181.

Mandel, J., 1964. The precision and accuracy of measurements. In: The Statistical Analysis of Experimental Data. Dover Publications, New York, pp. 102-130 (Chapter 6).

Melexis Data Sheet. MLX90614 family, single and dual zone infrared thermometer in TO-39. Rev. 005, March 30, 2009.

Omega Engineering, 2000. Infrared thermometers and pyrometers. Transactions in measurement and control, vol. I., 2nd ed. Putman Publishing Co. and Omega Press (Chapter 3).

O'Shaughnessy, S.A., Evett, S.R., 2007. IRT wireless interface for automatic irrigation scheduling of a center pivot system. In: Proc. 28th Annual International Irrigation Association Show. San Diego, Calif. pp. 176-186, Irrig. Assoc., Reston, VA.

O'Shaughnessy, S.A., Evett, S.R., 2010. Developing wireless sensor networks for monitoring crop canopy temperature using a moving sprinkler system as a platform. Appl. Eng. Agric. 26, 331-341.

Peters, R.T., Evett, S.R., 2004. Modeling diurnal canopy temperature dynamics using one-time-of-day measurements and a reference temperature curve. Agron. J. 96, 1553-1561.
Peters, R.T., Evett, S.R., 2007. Spatial and temporal analysis of crop stress using multiple canopy temperature maps created with an array of center-pivot-mounted infrared thermometers. Trans. ASABE 50, 919-927.

Pinter, P.J., Fry, K.E., Guinn, G., Mauney, J.R., 1983. Infrared thermometry: a remote sensing technique for predicting yield in water-stressed cotton. Agric. Water Manage. 6, 385-395.

Sadler, E.J., Camp, C.R., Evans, D.E., Millen, J.A., 2002. Spatial variation of corn response to irrigation. Trans. ASAE 45, 1869-1881.

Shimizu, Y., Ishii, J., 2008. Middle temperature scale for infrared radiation thermometer calibrated against multiple fixed points. Int. J. Thermophys. 29 1014-1025.

Taylor, J.R., 1997. An Introduction to Error Analysis, 2nd ed. University Science Books, Sausalito, Calif.

Wang, N.W., Zhang, N., Wang, M., 2006. Wireless sensor in agriculture and food industry - recent development and future perspective. Comput. Electron. Agric. 50 (1), 14.

Wanjura, D.F., Upchurch, D.R., 2001. Infrared thermometer calibration and viewing method effects on canopy temperature measurement. Agric. Forest Meteor. 55, 309-321. 